\title{
Using Symmetry to Count Rational Curves
}

\author{
Aaron Bertram! \\ Dedicated to Herb Clemens
}

The recent "close encounter" between enumerative algebraic geometry and theoretical physics has resulted in many new applications and new techniques for counting algebraic curves on a complex projective manifold. For example, string theorists demonstrated that generating functions built from counting curves can have completely unexpected relationships with other geometric constructions, via mirror symmetry [8]. In this paper, I want to focus on generating functions built from counting rational curves, and another insight inspired by physicists - the existence of hidden symmetries in the generating functions themselves.

By "counting rational curves," I mean producing numbers such as:

- the number of lines in $\mathbf{P}^{n}$ through two general points (1)

- the number of lines in $\mathbf{P}^{3}$ meeting 4 general lines (2)

- the number of rational quartics in $\mathbf{P}^{2}$ through 11 general points (620)

- the expected number of twisted cubic curves on a general quintic in $\mathbf{P}^{6}$ passing through 2 general points $(2,088,000)$

The first number is basic, the second is "classical", but the last two were not known until quite recently. There are now several methods for computing them [9, 10, 18], but the goal of this paper is to show how easily such computations follow from considerations of symmetry. More precisely, I will review WDVV symmetry and the reconstruction theorem of Kontsevich, and then introduce an analogous family of new symmetries and corresponding reconstruction theorem recently discovered in joint work together with Holger Kley [7].

Let $X$ be a complex projective manifold, and define:

$V=H^{*}(X, \mathbf{Q})$ with the Poincaré pairing $\gamma_{1} \otimes \gamma_{2} \mapsto \int_{X} \gamma_{1} \wedge \gamma_{2}$

$C_{\text {eff }} \subset H_{2}(X, \mathbf{Z})$, the set of positive linear combinations of classes $f_{*}[C]$, where $C$ is a Riemann surface and $f: C \rightarrow X$ is a morphism. Since $C_{\text {eff }}$ has the property that for each $\beta \in C_{\text {eff }}$ :

$$
C_{e f f} \cap\left(\beta-C_{e f f}\right)=\left\{\alpha \in C_{e f f} \mid \beta-\alpha \in C_{e f f}\right\} \text { is finite }
$$

it follows that the "Novikov ring" $\mathbf{Q}[[q]]$, which is the set of (infinite) sums $\sum_{\beta \in C_{e f f}} a_{\beta} q^{\beta}$ with $a_{\beta} \in \mathbf{Q}$, has a well-defined multiplication with $q^{\alpha} q^{\beta}=q^{\alpha+\beta}$.

All the generating functions will be defined in terms of $V$ and $\mathbf{Q}[[q]]$.

\footnotetext{
${ }^{1}$ Supported in part by NSF Research Grant DMS-9970412
} 
1. WDVV Symmetry. The Gromov-Witten invariant $\left\langle\gamma_{1}, \ldots, \gamma_{n}\right\rangle_{\beta}$, for given $\beta \in C_{\text {eff }}$ and $\gamma_{1}, \ldots, \gamma_{n} \in V$, counts the expected number of rational curves of class $\beta$ "meeting" the classes $\gamma_{i}$ (as in the earlier examples). We will show below how this definition is made rigorous by means of an explicit symmetric element $c_{0, n}^{\beta}(X) \in V^{\otimes n} \cong H^{*}\left(X^{n}, \mathbf{Q}\right)$ with the definition:

$$
\left\langle\gamma_{1}, \ldots, \gamma_{n}\right\rangle_{\beta}:=\int_{X^{n}} c_{0, n}^{\beta}(X) \wedge \gamma_{1} \otimes \ldots \otimes \gamma_{n}
$$

Think of $S y m^{*} V$ as the permutation-invariant sub-algebra of the tensor algebra, and define:

$$
c_{0}(X):=\sum_{\beta} \sum_{n \geq 3} c_{0, n}^{\beta}(X) q^{\beta} \in \operatorname{Sym}^{*} V[[q]]=\operatorname{Sym}^{*} V \otimes_{\mathbf{Q}} \mathbf{Q}[[q]]
$$

This starts with $n=3$ because of the impossibility of finding a good definition for the $c_{0, n}^{0}(X)$ for $n<3$ (but all $c_{0, n}^{\beta}(X)$ do have good definitions when $\beta \neq 0$ ).

So far, $c_{0}(X)$ is just a convenient means of packaging the Gromov-Witten invariants, but a key insight coming from string theory is the hidden:

WDVV Symmetry: For the contraction "]" defined below,

$$
\left.c_{0}(X)\right\rfloor c_{0}(X) \in \operatorname{Sym}^{*} V \otimes V^{\otimes 4}[[q]]
$$

is invariant under the permutation action of the symmetric group $S_{4}$ on $V^{\otimes 4}$.

The contraction is defined by partially desymmetrizing, thinking of:

$$
c_{0}(X) \in \operatorname{Sym}^{*} V \otimes V^{\otimes 3}[[q]]
$$

then isolating the last copy of $V$ from each $V^{\otimes 3}$ and contracting them via:

$$
V[[q]] \otimes V[[q]] \rightarrow \mathbf{Q}[[q]] ; \quad \gamma_{1} q^{\alpha_{1}} \otimes \gamma_{2} q^{\alpha_{2}} \mapsto\left(\int_{X} \gamma_{1} \wedge \gamma_{2}\right) q^{\alpha_{1}+\alpha_{2}},
$$

and finally partially symmetrizing via the map: $S y m^{*} V \otimes S y m^{*} V \rightarrow S y m^{*} V$.

Example: The coefficient of $q^{0}$ in $c_{0}(X)$ is the diagonal $\Delta_{3} \in V^{\otimes 3}$, and:

$$
\left.\Delta_{3}\right\rfloor \Delta_{3}=\Delta_{4} \in V^{\otimes 4}
$$

is symmetric. On the other hand, an arbitrary symmetric element of $V^{\otimes 3}$ does not result in a symmetric element of $V^{\otimes 4}$. For instance, if $X=\mathbf{P}^{1}$, then:

$$
\left.\left(1^{\otimes 3}+H^{\otimes 3}\right)\right\rfloor\left(1^{\otimes 3}+H^{\otimes 3}\right)=1^{\otimes 2} \otimes H^{\otimes 2}+H^{\otimes 2} \otimes 1^{\otimes 2}
$$

is symmetric under the subgroup of $S_{4}$ generated by (12),(34) and (13)(24) (These symmetries always hold.) The extra (2 3) symmetry is the novel one. 
At this point, a word is in order on the construction of the $c_{0, n}^{\beta}(X)$.

Definition: A pointed rational curve $p_{1}, \ldots, p_{n} \in C$ is stable if $C$ is a tree of smooth rational curves with nodes, the $p_{1}, \ldots, p_{n} \in C$ are distinct smooth points, and the automorphism group $\operatorname{Aut}\left(C ; p_{i}\right)$ of $C$, fixing the $p_{i}$, is finite.

Theorem: (Mumford-Knudsen) If $n \geq 3$, a fine moduli space of stable pointed rational curves exists. It is projective, smooth of dimension $n-3$, denoted $\bar{M}_{0, n}$.

Examples: $\bar{M}_{0,3}=$ point, $\bar{M}_{0,4} \cong \mathbf{P}^{1}$.

Inspired by this, Kontsevich-Manin made the following definition [14]:

Definition: A map $f:\left(C ; p_{1}, \ldots, p_{n}\right) \rightarrow X$ from a tree of smooth rational curves with distinct smooth points is stable if the automorphism group $\operatorname{Aut}\left(C ; p_{i} ; f\right)$ of $C$, fixing the $p_{i}$ and the map $f$, is finite.

Examples: All maps with finite fibers are stable. A constant map is stable if and only if the pointed curve is stable.

Theorem: (Kontsevich-Manin) A proper, Deligne-Mumfod stack of stable maps, denoted $\bar{M}_{0, n}(X, \beta)$, exists for each fixed $\beta$. It is smooth of dimension $\operatorname{dim}(X)-K_{X} \cap \beta+n-3$ when $X$ is nice enough (e.g. a homogeneous space).

Moreover, the moduli space comes equipped with "structure" maps:

$$
\begin{gathered}
\bar{M}_{0, n}(X, \beta) \stackrel{e v}{\rightarrow} X^{n} \\
\bar{M}_{0, n} \downarrow
\end{gathered}
$$

where $e v(f)=\left(f\left(p_{1}\right), \ldots, f\left(p_{n}\right)\right)$ and $\pi(f)$ is the "stabilization" of $\left(C ; p_{1}, \ldots, p_{n}\right)$. Of course, $\pi$ is only defined when $n \geq 3$. Finally, there is a diagram:

$$
\begin{gathered}
\bar{M}_{0, n+1}(X, \beta) \stackrel{e}{\rightarrow} X \\
f \downarrow \\
\bar{M}_{0, n}(X, \beta)
\end{gathered}
$$

where $f$ forgets the marked point $p_{n+1}$ and stabilizes, and $e(f)=f\left(p_{n+1}\right)$. This exhibits an isomorphism between $\bar{M}_{0, n+1}(X, \beta)$ and the "universal curve" $\mathcal{C}$.

When $X$ is a "nice enough" (see the theorem), then we define:

$$
c_{0, n}^{\beta}(X)=e v_{*}(1)
$$

Otherwise, the "right" definition for $c_{0, n}^{\beta}(X)$ has been found by Li-Tian and Behrend-Fantechi via the "virtual" fundamental class on $\bar{M}_{0, n}(X, \beta)$ [3, 16]. Here I will ignore these subtleties, referring to these classes only as $e v_{*}(1)$ and using the properties listed in [4] wherever convenient.

A couple of key relations among Gromov-Witten invariants follow: 
Basic Axioms: (a) (Easy String) $\left\langle\gamma_{1}, \ldots, \gamma_{n}, 1\right\rangle_{\beta}=0$ unless $n=2, \beta=0$.

(b) (Easy Divisor) If $D \in V$ is the class of a divisor on $X$, then

$$
\left\langle\gamma_{1}, \ldots, \gamma_{n}, D\right\rangle_{\beta}=(D \cap \beta)\left\langle\gamma_{1}, \ldots, \gamma_{n}\right\rangle_{\beta} \quad \text { unless } n=2, \beta=0
$$

Proof: Apply the projection formula to the commuting diagram:

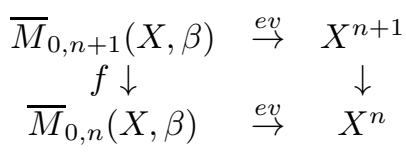

together with the fact that $f_{*} 1=0$ and $f_{*} e^{*} D=(D \cap \beta) \cdot 1$.

The proof of WDVV requires the additional map to $\bar{M}_{0, n}$. Given a subset $S \subseteq\{1, \ldots, n\}$ of cardinality $m$ and $\alpha \in C_{e f f}$, there is a gluing morphism:

$$
\delta_{S, \alpha}: \bar{M}_{0, m+1}(X, \alpha) \times_{X} \bar{M}_{0, n-m+1}(X, \beta-\alpha) \rightarrow \bar{M}_{0, n}(X, \beta)
$$

sending a pair of maps $f:\left(C ; p_{1}, \ldots, p_{m}, p\right) \rightarrow X$ and $g:\left(B ; q_{1}, \ldots, q_{n-m}, q\right) \rightarrow X$ satisfying $f(p)=g(q)$ to the single map $h:\left(C \cup_{p=q} B ; p_{i}, q_{j}\right) \rightarrow X$. To give the ordering of the points on $C \cup B$, apply the permutation $\sigma_{S}$ to $\left\{p_{i}, q_{j}\right\}$ given by:

$\sigma_{S}(i)=s_{i}, \sigma_{S}(m+i)=s_{i}^{c}$ where $S=\left\{s_{1}<\ldots<s_{m}\right\}, S^{c}=\left\{s_{1}^{c}<\ldots<s_{n-m}^{c}\right\}$

The gluing maps have two important properties:

- $e v_{*} \delta_{S, \alpha_{*}}(1)=\sigma_{S}\left(c_{0, m+1}^{\alpha} \mid c_{0, n-m+1}^{\beta-\alpha}\right)$ where $\sigma_{S}$ permutes the factors of $V^{\otimes n}$.

- $\pi^{*} \delta_{S *}(1)=\sum_{\alpha} \delta_{S, \alpha_{*}}(1)$ for $\delta_{S}: \bar{M}_{0, m+1} \times \bar{M}_{0, n-m+1} \rightarrow \bar{M}_{0, n}$.

In addition, one easily checks that:

- $c r^{*} \delta_{\{i j\}_{*}}(1)=\sum_{S} \delta_{S \cup\{n+i, n+j\}_{*}}(1)$ for $i, j \leq 4$ and the forgetful cross-ratio map $c r: \bar{M}_{0, n+4} \rightarrow \bar{M}_{0,4}$ But $\delta_{\{i j\}_{*}}(1)$ is the class of a point on $\bar{M}_{0,4}=\mathbf{P}^{1}$, so is independent of $i, j$, and all together, we get:

$$
\begin{aligned}
& \left.\sum_{m} \sum_{|S|=m} \sum_{\alpha} \sigma_{S \cup\{n+1, n+2\}}\left(c_{0, m+3}^{\alpha}\right\rfloor c_{0, n-m+3}^{\beta-\alpha}\right)=e v_{*} \pi^{*} c r^{*} \delta_{\{12\}_{*}}(1)= \\
& \left.=e v_{*} \pi^{*} c r^{*} \delta_{\{13\}_{*}}(1)=\sum_{m} \sum_{|S|=m} \sum_{\alpha} \sigma_{S \cup\{n+1, n+3\}}\left(c_{0, m+3}^{\alpha}\right\rfloor c_{0, n-m+3}^{\beta-\alpha}\right)
\end{aligned}
$$

which are precisely the coefficients of $q^{\beta}$ in $\left.c_{0}(X)\right\rfloor c_{0}(X)$ and the pull-back of $\left.c_{0}(X)\right\rfloor c_{0}(X)$ under the (2 3) transposition, respectively.

Remark: Checking these properties of the gluing maps with virtual classes is the major technical point in the work of Behrend-Fantechi and Li-Tian. 
2. Kontsevich Reconstruction. This is generally understood to be the following consequence of the WDVV relations (and the basic axioms):

Theorem: (Kontsevich [14]) If $V$ is generated by classes of divisors, then $c_{0}(X)$ can be explicitly reconstructed from the $c_{0,2}^{\beta}(X)$ and the class $c_{0,3}^{0}(X)=\Delta_{3}$.

Proof: Choose generating divisor classes $D_{1}, \ldots, D_{k}$, and choose a basis $\left\{D_{J}=\prod D_{i}^{j_{i}}\right\} \in V$ consisting of monomials in the divisors $D_{j}$. For $n \geq 0, \beta>0$ and $|K| \geq 2$, we use WDVV to solve for Gromov-Witten invariants of the form:

$$
\left\langle\gamma_{1}, \ldots, \gamma_{n+2}, D_{K}\right\rangle_{\beta}
$$

in terms of invariants involving either:

(i) smaller $\beta$ (and either the same or smaller $n$ ) or

(ii) the same $\beta$ and $n$ but smaller $|K|$.

Since the basic axioms allow us to eliminate $D_{K}$ in case $|K|=0$ or 1 , the theorem then follows by induction.

Let $\left(g^{I J}\right)$ be the inverse of the intersection matrix defined by:

$$
g_{I J}:=\int_{X} D_{I} \wedge D_{J}
$$

and choose $D_{L}, D_{M}$ so that $D_{L} \wedge D_{M}=D_{K}$ with $|L|,|M|<|K|$. Then the coefficient of $q^{\beta}$ in the expression:

$$
\left.\int_{X^{n+4}} c_{0}(X)\right\rfloor c_{0}(X) \wedge \gamma_{1} \otimes \ldots \otimes \gamma_{n+2} \otimes D_{L} \otimes D_{M}
$$

is, explicitly,

(1) $\sum_{S} \sum_{I, J}\left\langle\gamma_{s_{1}}, \ldots, \gamma_{s_{m}}, \gamma_{n+1}, \gamma_{n+2}, D_{I}\right\rangle_{\alpha} g^{I J}\left\langle D_{J}, \gamma_{s_{1}^{c}}, \ldots, \gamma_{s_{n-m}^{c}}, D_{L}, D_{M}\right\rangle_{\beta-\alpha}$

whereas after the (2 3) permutation, we obtain:

(2) $\sum_{S} \sum_{I, J}\left\langle\gamma_{s_{1}}, \ldots, \gamma_{s_{m}}, \gamma_{n+1}, D_{L}, D_{I}\right\rangle_{\alpha} g^{I J}\left\langle D_{J}, \gamma_{s_{1}^{c}}, \ldots, \gamma_{s_{n-m}^{c}}, \gamma_{n+2}, D_{M}\right\rangle_{\beta-\alpha}$

Only the terms $\alpha=0, S=\emptyset$ or $\alpha=\beta, S=\{1, \ldots, n\}$ do not satisfy $(i)$. But these terms can be computed using the fact that $c_{0,3}^{0}(X)=\Delta_{3}$. They are:

(1) $\left\langle\gamma_{n+1} \wedge \gamma_{n+2}, \gamma_{1}, \ldots, \gamma_{n}, D_{L}, D_{M}\right\rangle_{\beta}+\left\langle\gamma_{1}, \ldots, \gamma_{n+2}, D_{K}\right\rangle_{\beta}$ and

(2) $\left\langle\gamma_{n+1} \wedge D_{L}, \gamma_{1}, \ldots, \gamma_{n}, \gamma_{n+2}, D_{M}\right\rangle_{\beta}+\left\langle\gamma_{1}, \ldots, \gamma_{n+1}, D_{L}, \gamma_{n+2} \wedge D_{M}\right\rangle_{\beta}$

The theorem follows since we are solving for the second of the terms in (1) and the other three of these "special" terms satisfy (ii). 
Examples: (a) For $X=\mathbf{P}^{3}$ and $\beta=1$, there are only the special terms, and taking $\gamma_{1}=H^{2}, \gamma_{2}=H^{2}, \gamma_{3}=H^{2}, D_{L}=H, D_{M}=H$ gives:

$$
\left\langle H^{4}, H^{2}, H, H\right\rangle_{1}+\left\langle H^{2}, H^{2}, H^{2}, H^{2}\right\rangle_{1}=\left\langle H^{3}, H^{2}, H^{2}, H\right\rangle_{1}+\left\langle H^{2}, H^{2}, H, H^{3}\right\rangle_{1}
$$

from which one concludes using the divisor axiom that:

$$
\left\langle H^{2}, H^{2}, H^{2}, H^{2}\right\rangle_{1}=2\left\langle H^{3}, H^{2}, H^{2}\right\rangle_{1}
$$

and letting $\gamma_{1}=H^{3}, \gamma_{2}=H^{2}, D_{L}=H, D_{M}=H$ gives:

$$
\left\langle H^{5}, H, H\right\rangle_{1}+\left\langle H^{3}, H^{2}, H^{2}\right\rangle_{1}=\left\langle H^{4}, H^{2}, H\right\rangle_{1}+\left\langle H^{3}, H, H^{3}\right\rangle_{1}
$$

which gives $\left\langle H^{3}, H^{2}, H^{2}\right\rangle_{1}=\left\langle H^{3}, H^{3}\right\rangle_{1}=1$ and $\left\langle H^{2}, H^{2}, H^{2}, H^{2}\right\rangle_{1}=2$.

(b) For a more interesting example, let let $n_{d}$ be the number of rational plane curves of degree $d$ through $3 d-1$ general points of $\mathbf{P}^{2}$. That is,

$$
n_{d}=\left\langle H^{2}, \ldots, H^{2}\right\rangle_{d}
$$

Let $\gamma_{1}, \ldots, \gamma_{3 d-2}=H^{2}$ and $D_{L}=D_{M}=H$. Then:

(1) $0+n_{d}+\sum_{0<e<d} \sum_{|S|=3 e-3}\left\langle H^{2}, \ldots, H^{2}, H^{2}, H^{2}, H\right\rangle_{e}\left\langle H, H^{2}, \ldots, H^{2}, H, H\right\rangle_{d-e}$

(2) $0+0+\sum_{0<e<d} \sum_{|S|=3 e-2}\left\langle H^{2}, \ldots, H^{2}, H^{2}, H, H\right\rangle_{e}\left\langle H, H^{2}, \ldots, H^{2}, H^{2}, H\right\rangle_{d-e}$

and this, together with the divisor equation, gives the recursive formula:

$$
n_{d}=\sum_{e=1}^{d-1} n_{e} n_{d-e}\left(e^{2}(d-e)^{2}\left(\begin{array}{c}
3 d-4 \\
3 e-2
\end{array}\right)-e(d-e)^{3}\left(\begin{array}{c}
3 d-4 \\
3 e-3
\end{array}\right)\right)
$$

from which (together with the a priori $n_{1}=1$ ) one computes:

$$
n_{2}=1, n_{3}=12, n_{4}=620 \text { (as in the introduction) }
$$

Remarks: The reconstruction theorem applies in a more general situation. If we can write $V=W+W^{\perp}$ so that $W$ is generated by divisor classes and every Gromov-Witten invariant invariant of the form $\left\langle\gamma_{1}, \ldots, \gamma_{n}, \lambda\right\rangle_{\beta}$ is zero when $\gamma_{1}, \ldots, \gamma_{n} \in W$ and $\lambda \in W^{\perp}$, then every Gromov-Witten invariant of the form $\left\langle\gamma_{1}, \ldots, \gamma_{n}\right\rangle_{\beta}$ (for all $\gamma_{i} \in W$ ) can be reconstructed from the $n=2$ invariants. The same proof gives this result, as well.

This applies, for example, to the case of a hypersurface $X \subset \mathbf{P}^{n}$. Which begs the question: What are the $c_{0,2}^{\beta}(X)$ in that case? Holger Kley and I found a way to reconstruct these, too, using other hidden symmetries. 
3. A Symmetry of $J$-functions. The universal curve:

$$
\begin{gathered}
\mathcal{C} \\
f \downarrow \\
\bar{M}_{0, n}(X, \beta)
\end{gathered}
$$

with universal sections (the marked points) $\rho_{i}: \bar{M}_{0, n}(X, \beta) \rightarrow \mathcal{C}$ determine the "Morita classes" $\psi_{i}:=c_{1}\left(N_{\rho_{i}}^{*}\right)$, where $N_{\rho_{i}}^{*}$ is the conormal bundle of the section. The following strange-looking definition is inspired by mirror symmetry (as interpreted by Givental [11], Lian-Liu-Yau [17] and others):

$$
J_{0, n}^{\beta}(X):=e v_{*}\left(\frac{1}{\prod_{i=1}^{n} t_{i}\left(t_{i}-\psi_{i}\right)}\right) \in \operatorname{Sym}^{n}\left(V\left[t^{-1}\right]\right)
$$

where $t$ (or rather $t_{i}$ ) is a variable, and the denominator is formally inverted. Note that the coefficient of $\prod t_{i}^{-2}$ is $c_{0, n}^{\beta}(X)$.

These seem to have better properties than the $c_{0, n}^{\beta}(X)$. For example, the $n=1$ (mirror conjecture) case has some very interesting "functorial" properties and is often amenable to computations.

Theorem: (Bertram/Behrend [5, 2) If $\beta \in C_{\text {eff }}(X \times Y)$, let $\beta_{1} \in C_{\text {eff }}(X)$ and $\beta_{2} \in C_{e f f}(Y)$ be the two projections. Then:

$$
J_{0,1}^{\beta}(X \times Y)=J_{0,1}^{\beta_{1}}(X) \otimes_{\mathbf{Q}\left[t^{-1}\right]} J_{0,1}^{\beta_{2}}(Y)
$$

(there is a product formula for $c_{0,2}^{\beta}(X)$ classes, but it is much more complex)

Theorem: (Givental [11) Let $H$ be the hyperplane class in $H^{2}\left(\mathbf{P}^{n}, \mathbf{Z}\right)$. Then:

$$
J_{0,1}^{d}\left(\mathbf{P}^{n}\right)=\frac{1}{\prod_{k=1}^{d}(H+k t)^{n+1}}
$$

If $X \subset \mathbf{P}^{n}$ is a complete intersection of type $\left(l_{1}, \ldots, l_{r}\right)$ of dimension $\geq 3$ with $l_{1}+\ldots+l_{r}<n$, then:

$$
J_{0,1}^{d}(X)=\frac{\prod_{i=1}^{r} \prod_{k=1}^{d l_{i}}\left(l_{i} H+k t\right)}{\prod_{k=1}^{d}(H+k t)^{n+1}}
$$

and if $l_{1}+\ldots+l_{r}=n$ or $n+1$, then $J_{0,1}^{d}(X)$ is computed from the $J_{0,1}^{d}\left(\mathbf{P}^{n}\right)$ by an explicit "mirror transformation" (see [6]).

This generalizes to complete intersections in (Fano) toric varieties, but it is even more general, as pointed out first by Kim and proved by Lee [15]:

Quantum Lefschetz Hyperplane Theorem: If $X \subset Y$ is a Fano or CalabiYau very ample hypersurface of dimension at least 3 , then the $J_{0,1}^{\beta}(X)$ are explicitly determined by the $J_{0,1}^{\beta}(Y)$. 
The following computation:

$$
J_{0, n}^{0}(X)=\Delta_{n} \prod t_{i}^{-2}\left(\sum t_{i}^{-1}\right)^{n-3}
$$

follows from the well-known intersection numbers on $\bar{M}_{0, n}$ :

$$
\int_{\bar{M}_{0, n}} \psi_{1}^{a_{1}} \wedge \ldots \wedge \psi_{n}^{a_{n}}=\frac{(n-3) !}{a_{1} ! \ldots a_{n} !}
$$

whenever $n \geq 3$ and $\sum a_{i}=n-3$. We argue by analogy that:

$$
J_{0,1}^{0}(X)=1 \text { and } \quad J_{0,2}^{0}(X)=\frac{\Delta_{2}}{t_{1} t_{2}\left(t_{1}+t_{2}\right)}
$$

are good definitions (though the second one does not belong to $S y m^{2}\left(V\left[t^{-1}\right]\right)$ ).

With these definitions, we put together the generating function:

$$
J_{0}(X):=\sum_{\beta} \sum_{n \geq 1} J_{0, n}^{\beta}(X) q^{\beta} \in \operatorname{Sym}^{*}\left(V\left[t^{-1}\right]\right)[[q]]
$$

(with a little extra room for $J_{0,2}^{0}(X)$ ), and by analogy with WDVV, we have:

Theorem 1: (Bertram-Kley [7]) The $J_{0}(X)$ invariants have the symmetry:

$$
\left.J_{0}(X)\right\rfloor J_{0}(X)=0
$$

defined as follows. Let $U=V\left[t^{-1}\right]$ and extend Poincaré duality to:

$U[[q]] \otimes U[[q]] \rightarrow \mathbf{Q}\left[t^{-1}\right][[q]] ; \gamma_{1} t^{-k} q^{\alpha} \otimes \gamma_{2} t^{-l} q^{\beta} \mapsto\left(\int_{X} \gamma_{1} \wedge \gamma_{2}\right) t^{-k}(-t)^{-l} q^{\alpha+\beta}$

Partially desymmetrize, writing $J_{0}(X)$ as an element of $\operatorname{Sym}^{*}(U) \otimes U[[q]]$, and:

$$
\left.J_{0}(X)\right\rfloor J_{0}(X)=0 \in \operatorname{Sym}^{*}(U)\left[t^{-1}\right][[q]]
$$

is a collection of identities on the coefficients of the $t^{-k}$.

The Extra Term: Contracting with $J_{0,2}^{0}(X)$ does not fit the pattern above, at least not in the obvious way. Instead, we extend the Poincaré duality to:

$$
\gamma_{1} t^{-k} \otimes \frac{\gamma_{2} t^{-l}}{t_{i}+t} \mapsto\left(\int_{X} \gamma_{1} \wedge \gamma_{2}\right) \frac{t^{-k}-t_{i}^{-k}}{t_{i}-t}(-t)^{-l}
$$

so that $J_{0,2}^{0}(X)$ acts as a difference operator, and similarly,

$$
\frac{\gamma_{1} t^{-k}}{t_{i}+t} \otimes \gamma_{2} t^{-l} \mapsto\left(\int_{X} \gamma_{1} \wedge \gamma_{2}\right) \frac{(-t)^{-l}-t_{i}^{-l}}{t_{i}+t} t^{-k}
$$


As an example, we use Theorem 1 to prove the:

String Equation: Define the generalized Gromov-Witten invariants by:

$$
\left\langle\psi^{a_{1}}\left(\gamma_{1}\right), \ldots, \psi^{a_{n}}\left(\gamma_{n}\right)\right\rangle_{\beta}:=\int_{X^{n}} e v_{*}\left(\psi_{1}^{a_{1}} \ldots \psi_{n}^{a_{n}}\right) \wedge \gamma_{1} \otimes \ldots \otimes \gamma_{n}
$$

if each $a_{i} \geq 0$, and zero if some $a_{i}<0$. Then unless $n=1$ or 2 and $\beta=0$,

$$
\left\langle\psi^{a_{1}}\left(\gamma_{1}\right), \ldots, \psi^{a_{n}}\left(\gamma_{n}\right), 1\right\rangle_{\beta}=\sum_{i=1}^{n}\left\langle\psi^{a_{1}}\left(\gamma_{1}\right), \ldots, \psi^{a_{i}-1}\left(\gamma_{i}\right), \ldots, \psi^{a_{n}}\left(\gamma_{n}\right)\right\rangle_{\beta}
$$

Proof: The $U^{\otimes n} q^{\beta}$ coefficient of $\left.J_{0}(X)\right\rfloor J_{0}(X)$ is:

$$
\left.\sum_{\alpha} \sum_{m} \sum_{|S|=m} \sigma_{S}\left(J_{0, m+1}^{\alpha}(X)\right\rfloor J_{0, n-m+1}^{\beta-\alpha}(X)\right)
$$

and we say that a summand involving $J_{0,1}^{0}(X)$ or $J_{0,2}^{0}(X)$ is special. Let $\left\{e_{i}\right\}$ be a basis of $V$ with intersection matrix $g_{i j}$, and consider the $q^{\beta}$ coefficient of:

$$
\left.\int_{X^{n}} J_{0}(X)\right\rfloor J_{0}(X) \wedge \gamma_{1} \otimes \ldots \otimes \gamma_{n}
$$

The non-special summands produce terms of the form:

$$
\sigma_{S}\left\langle\frac{\gamma_{1}}{t_{1}\left(t_{1}-\psi\right)}, \ldots, \frac{e_{i}}{t(t-\psi)}\right\rangle_{\alpha} g^{i j}\left\langle\frac{e_{j}}{-t(-t-\psi)}, \ldots, \frac{\gamma_{n}}{-t_{n}\left(-t_{n}-\psi\right)}\right\rangle_{\beta-\alpha}
$$

where we formally set $\frac{\gamma}{t(t-\psi)}=t^{-2} \gamma+t^{-3} \psi(\gamma)+t^{-4} \psi^{2}(\gamma)+\ldots$

On the other hand, the special summands produce:

$$
\begin{gathered}
(m=n, \alpha=\beta) \quad\left\langle\frac{\gamma_{1}}{t_{1}\left(t_{1}-\psi\right)}, \ldots, \frac{\gamma_{n}}{t_{n}\left(t_{n}-\psi\right)}, \frac{1}{t(t-\psi)}\right\rangle_{\beta} \quad \text { and } \\
(m=n-1, \alpha=\beta) \quad \sum_{i=1}^{n}\left\langle\frac{\gamma_{1}}{t_{1}\left(t_{1}-\psi\right)}, \ldots, f_{t, t_{i}}\left(\gamma_{i}\right), \ldots, \frac{\gamma_{n}}{t_{n}\left(t_{n}-\psi\right)}\right\rangle_{\beta}
\end{gathered}
$$

where

$$
f_{t, t_{i}}=\frac{(t(t-\psi))^{-1}-\left(t_{i}\left(t_{i}-\psi\right)\right)^{-1}}{t_{i}(-t)\left(t_{i}-t\right)}
$$

and the $\alpha=0$ terms, which are the same functions, but of $-t$ instead of $t$.

Setting the coefficient of $t^{-2}$ to zero (using Theorem 1) gives:

$$
\left\langle\frac{\gamma_{1}}{t_{1}\left(t_{1}-\psi\right)}, . ., \frac{\gamma_{n}}{t_{n}\left(t_{n}-\psi\right)}, 1\right\rangle_{\beta}=\sum_{i=1}^{n}\left\langle\frac{\gamma_{1}}{t_{1}\left(t_{1}-\psi\right)}, \ldots, \frac{\gamma_{i}}{t_{i}^{2}\left(t_{i}-\psi\right)}, \ldots, \frac{\gamma_{n}}{t_{n}\left(t_{n}-\psi\right)}\right\rangle_{\beta}
$$

which is the string equation! 
4. More Symmetries of $J$-Functions. Theorem 1 is a consequence of a family of symmetries of $J$-functions, which will occupy the rest of this paper.

Definition: Let $J_{0}^{\prime}(X) \in S y m^{*} U \otimes U^{\otimes 2}[[q]]$ be the desymmetrization of $J_{0}(X)$.

(this notation distinguishes it from $J_{0}(X) \in S y m^{*} U \otimes U[[q]]$ )

Theorem 2: (Bertram-Kley [7])

$$
\left.J_{0}^{\prime}(X)\right\rfloor J_{0}(X)=0 \in \operatorname{Sym}^{*} U \otimes U\left[t^{-1}\right][[q]]
$$

There are more symmetries, but first I want to give a:

Stringy Corollary: Every (generalized) Gromov-Witten invariant of the form:

$$
\left\langle\psi^{a_{0}}\left(\gamma_{0}\right), \psi^{a_{1}}\left(\gamma_{1}\right), \ldots, \psi^{a_{n}}\left(\gamma_{n}\right), \psi^{a_{n+1}}(1)\right\rangle_{\beta}
$$

can be explicitly expressed in terms of "simpler" invariants.

Consider the $q^{\beta}$ coefficient of:

$$
\left.\int_{X^{n+1}} J_{0}^{\prime}(X)\right\rfloor J_{0}(X) \wedge \gamma_{0} \otimes \ldots \otimes \gamma_{n}
$$

(where we index the distinguished factor of $S y m^{*} U \otimes U$ with the zero subscript).

This consists of special terms:

$$
\begin{aligned}
\left\langle\frac{\gamma_{0}}{t_{0}\left(t_{0}-\psi\right)}, \ldots,\right. & \left.\frac{\gamma_{n}}{t_{n}\left(t_{n}-\psi\right)}, \frac{1}{t(t-\psi)}\right\rangle_{\beta}+\left\langle f_{-t, t_{0}}\left(\gamma_{0}\right), \frac{\gamma_{1}}{t_{1}\left(t_{1}-\psi\right)} \ldots, \frac{\gamma_{n}}{t_{n}\left(t_{n}-\psi\right)}\right\rangle_{\beta} \\
& +\sum_{i=1}^{n}\left\langle\frac{\gamma_{0}}{t_{0}\left(t_{0}-\psi\right)}, \ldots, f_{t, t_{i}}\left(\gamma_{i}\right), \frac{\gamma_{n}}{t_{n}\left(t_{n}-\psi\right)}, \frac{1}{t(t-\psi)}\right\rangle_{\beta}
\end{aligned}
$$

where $f_{t, t_{i}}$ is the divided difference of $\left(t_{i}\left(t_{i}-\psi\right)\right)^{-1}$ defined earlier.

In addition, there are the non-special terms, of the form:

$$
\sigma_{\{0\} \cup S}\left\langle\frac{\gamma_{0}}{t_{0}\left(t_{0}-\psi\right)}, \ldots, \frac{e_{i}}{t(t-\psi)}\right\rangle_{\alpha} g^{i j}\left\langle\frac{e_{j}}{-t(-t-\psi)},, \ldots, \frac{\gamma_{n}}{-t_{n}\left(-t_{n}-\psi\right)}\right\rangle_{\beta-\alpha}
$$

But Theorem 2 says that the sum of all the terms is zero. So since the first special term generates all the Gromov-Witten invariants of the corollary, and all the other terms are simpler, the corollary is proved.

Examples: As before, we get the string equation from the coefficient of $t^{-2}$. From the coefficient of $t^{-3}$, we obtain the Dilaton Equation:

$$
\left\langle\psi^{a_{0}}\left(\gamma_{0}\right), \ldots, \psi^{a_{n}}\left(\gamma_{n}\right), \psi(1)\right\rangle_{\beta}=(n-1)\left\langle\psi^{a_{0}}\left(\gamma_{0}\right), \ldots, \psi^{a_{n}}\left(\gamma_{n}\right)\right\rangle_{\beta}
$$


Fix divisor classes $H_{1}, \ldots, H_{n} \in V$ and a polynomial $p\left(x_{1}, \ldots, x_{n}\right)$. For each $\beta \in C_{\text {eff }}$, let $b_{i}=H_{i} \cap \beta$, and define:

$$
p(H-\beta t)=p\left(H_{1}-b_{1} t, \ldots, H_{n}-b_{n} t\right)
$$

Then we have the following:

Theorem 3: (Bertram-Kley [7) For any polynomial $p$ as above:

$$
\left.J_{0}^{\prime}(X)\right\rfloor_{p} J_{0}(X) \in \operatorname{Sym}^{*}(U) \otimes U[[q]] \otimes t^{-1} \mathbf{Q}[t]
$$

where " $\rfloor_{p}$ " is the contraction defined using the pairing:

$$
\gamma_{1} t^{-k} q^{\alpha} \otimes \gamma_{2} t^{-l} q^{\beta} \mapsto\left(\int_{X} \gamma_{1} \wedge \gamma_{2} \wedge p(H-\beta t)\right) t^{-k}(-t)^{-l} \in \mathbf{Q}\left[t, t^{-1}\right] q^{\alpha+\beta}
$$

Remark: Theorem $3 \Rightarrow$ Theorem $2(\Rightarrow$ Theorem 1$)$ taking $p=1$.

Example: The Divisor Equation is obtained from $p(x)=x$. The special terms of:

$$
\left.\int_{X^{n+1}} J_{0}^{\prime}(X)\right\rfloor_{x} J_{0}(X) \wedge \gamma_{0} \otimes \ldots \otimes \gamma_{n}
$$

are:

$$
\begin{gathered}
\left\langle\frac{\gamma_{0}}{t_{0}\left(t_{0}-\psi\right)}, \ldots, \frac{H}{t(t-\psi)}\right\rangle_{\beta}+\left\langle f_{-t, t_{0}}\left(\gamma_{0} \wedge(H-b t)\right), \frac{\gamma_{1}}{t_{1}\left(t_{1}-\psi\right)} \ldots, \frac{\gamma_{n}}{t_{n}\left(t_{n}-\psi\right)}\right\rangle_{\beta} \\
+\sum_{i=1}^{n}\left\langle\frac{\gamma_{0}}{t_{0}\left(t_{0}-\psi\right)}, \ldots, f_{t, t_{i}}\left(\gamma_{i} \wedge H\right), \frac{\gamma_{n}}{t_{n}\left(t_{n}-\psi\right)}, \frac{1}{t(t-\psi)}\right\rangle_{\beta}
\end{gathered}
$$

and non-special terms do not contribute to the coefficient of $t^{-2}$, which gives:

$$
\begin{gathered}
\left\langle\psi^{a_{0}}\left(\gamma_{0}\right), \ldots, \psi^{a_{n}}\left(\gamma_{n}\right), H\right\rangle_{\beta}=b\left\langle\psi^{a_{0}}\left(\gamma_{0}\right), \ldots, \psi^{a_{n}}\left(\gamma_{n}\right)\right\rangle_{\beta} \\
+\sum_{i=0}^{n}\left\langle\psi^{a_{0}}\left(\gamma_{0}\right), \ldots, \psi^{a_{i}-1}\left(\gamma_{i} \wedge H\right), \ldots, \psi^{a_{n}}\left(\gamma_{n}\right)\right\rangle_{\beta}
\end{gathered}
$$

Reconstruction Corollary: If $V$ is generated by divisor classes, then $J_{0}(X)$ can be explicitly reconstructed from the $J_{0,1}^{\beta}(X)$ classes.

Proof: When we pair $\left.J_{0}^{\prime}(X)\right\rfloor_{p} J_{0}(X)$ with $\gamma_{0} \otimes \ldots \otimes \gamma_{n}$, then the first special term is of the form:

$$
\left\langle\frac{\gamma_{0}}{t_{0}\left(t_{0}-\psi\right)}, \ldots, \frac{\gamma_{n}}{t_{n}\left(t_{n}-\psi\right)}, \frac{p(H)}{t(t-\psi)}\right\rangle_{\beta}
$$

and all the other terms are "simpler." Since this only involves powers $t^{-2}, t^{-3}, \ldots$, it is inductively determined by the $J_{0,1}^{\beta}(X)$ using Theorem 3 and the fact that $V$ is generated by divisor classes. 
Remark: As with Kontsevich reconstruction, this corollary holds in greater generality. Let $W \subset V$ be the subalgebra generated by divisor classes. If the coefficients of $J_{0,1}^{\beta}(X)$ all belong to $W$, then all generalized invariants involving only classes coming from $W$ (i.e. the projection of $J_{0}(X)$ to $\left.\operatorname{Sym}^{*}\left(W\left[t^{-1}\right]\right)[[q]]\right)$ can be explicitly reconstructed from the $J_{0,1}^{\beta}(X)$.

If we only consider the $\prod t_{i}^{-2}$ terms, then by Theorem 3 , the coefficients of $t^{-2}, t^{-3}, \ldots$ vanish in each of the following expressions:

$$
\begin{aligned}
& \left\langle\gamma_{0}, \ldots, \gamma_{n}, \frac{p(H)}{t(t-\psi)}\right\rangle_{\beta}+\sum_{i=1}^{n}\left\langle\gamma_{0}, . ., \frac{\gamma_{i} \wedge p(H)}{-t^{2}(t-\psi)}, . ., \gamma_{n}\right\rangle_{\beta}+\left\langle\frac{\gamma_{0} \wedge p(H-b t)}{-t^{2}(-t-\psi)}, \ldots, \gamma_{n}\right\rangle_{\beta} \\
& +\sum_{\alpha} \sum_{m} \sum_{|S|=m} \sigma_{\{0\} \cup S}\left\langle\gamma_{0}, \ldots, \gamma_{m}, \frac{e_{i}}{t(t-\psi)}\right\rangle_{\beta-\alpha} g^{i j}\left\langle\frac{e_{j} \wedge p(H-a t)}{-t(-t-\psi)}, \gamma_{m+1}, \ldots, \gamma_{n}\right\rangle_{\alpha}
\end{aligned}
$$

and this leads to very efficient algorithms for reconstructing ordinary invariants, provided $J_{0,1}^{\beta}(X)$ are known and the conditions of the remark above are satisfied. Note that in order to reconstruct ordinary invariants, we can stay within the realm of invariants with at most one "gravitational descendant" $\psi^{a}$.

Example: Another look at rational plane curves. Let $X=\mathbf{P}^{2}$ and:

$$
\left.n_{d}^{(a)}:=\left\langle H^{2}, \ldots, H^{2}, \psi^{a}\left(H^{2}\right)\right\rangle_{d} \quad \text { (the total number of terms is } 3 d-1-a\right)
$$

Then it follows from our relations that:

$$
\begin{array}{ll}
n_{d} & =d^{2} n_{d}^{(1)}-\sum_{e=1}^{d-1}\left(\begin{array}{c}
3 d-3 \\
3 e-1
\end{array}\right)(d-e) e^{3} n_{d-e} n_{e} \\
n_{d}^{(1)} & =d^{2} n_{d}^{(2)}-\sum_{e=1}^{d-1}\left(\begin{array}{c}
3 d-4 \\
3 e-1
\end{array}\right)(d-e) e^{3} n_{d-e}^{(1)} n_{e}-\sum_{e=1}^{d-1}\left(\begin{array}{c}
3 d-4 \\
3 e-2
\end{array}\right) e^{2} n_{d-e} n_{e} \\
n_{d}^{(2)} & =d^{2} n_{d}^{(3)}-\sum_{e=1}^{d-1}\left(\begin{array}{c}
3 d-5 \\
3 e-1
\end{array}\right)(d-e) e^{3} n_{d-e}^{(2)} n_{e}-\sum_{e=1}^{d-1}\left(\begin{array}{c}
3 d-5 \\
3 e-2
\end{array}\right) e^{2} n_{d-e}^{(1)} n_{e} \\
\vdots & \\
n_{d}^{(3 d-3)} & =d^{2} n_{d}^{(3 d-2)}=\frac{1}{d(d-1 !)^{3}} \text { (from Givental) }
\end{array}
$$

In case $d \leq 3$, this gives: $n_{1}=n_{1}^{(1)}=1 ; n_{2}=n_{2}^{(1)}=n_{2}^{(2)}=1, n_{2}^{(3)}=\frac{1}{2}, n_{2}^{(4)}=\frac{1}{8}$;

$$
n_{3}=12, n_{3}^{(1)}=10, n_{3}^{(2)}=7, n_{3}^{(3)}=3, n_{3}^{(4)}=1, n_{3}^{(5)}=\frac{1}{4}, n_{3}^{(6)}=\frac{1}{24}, n_{3}^{(7)}=\frac{1}{216}
$$

It is amusing to note that $d=1$ "proves" there is one line through 2 points. But seriously, the "mirror data" $J_{0,1}^{\beta}(X)$ does appear in general to be more basic and a better starting point for inductions than the two-point invariants $c_{0,2}^{\beta}(X)$. On the other hand, if one is only interested in ordinary invariants, it is probably more efficient to compute the $c_{0,2}^{\beta}(X)$ first and then to apply Kontsevich reconstruction. This is easy to implement as an algorithm when $X$ is a Fano hypersurface in $\mathbf{P}^{n}$ (see the appendix in [7]). 
5. Localization. The proof of Theorem 3 relies on the localization theorem of Atiyah-Bott, as adapted by Graber and Pandharipande [12] to apply to virtual classes on the moduli stacks of stable maps. The idea of using localization to compute Gromov-Witten invariants was introduced by Kontsevich 13], but the key insight, described below, belongs to Givental [11].

Recall that in order to prove WDVV, one considered the map:

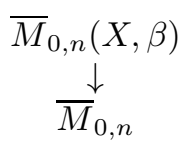

and relations in $H^{*}\left(\bar{M}_{0, n}, \mathbf{Q}\right)$ gave relations among Gromov-Witten invariants.

Here, the key insight is to consider the embedding:

$$
i: \bar{M}_{0, n}(X, \beta) \hookrightarrow \bar{M}_{0,0}\left(X \times\left(\mathbf{P}^{1}\right)^{n},\left(\beta, 1^{n}\right)\right)
$$

replacing each marked point with a curve mapping isomorphically to $\mathbf{P}^{1}$, with the node mapping to 0 (see [6]). In this way, $\bar{M}_{0, n}(X, \beta)$ is one of the (many!) connected components of the locus of fixed points for the natural action of the torus $T=\left(\mathbf{C}^{*}\right)^{n}$ on the "graph space" $\bar{M}_{0,0}\left(X \times\left(\mathbf{P}^{1}\right)^{n},\left(\beta, 1^{n}\right)\right)$.

The localization theorem says that an equivariant cohomology (or Chern) class $c$ on the graph space can be recovered from the total fixed-point locus $i: F \hookrightarrow \bar{M}_{0,0}\left(X \times\left(\mathbf{P}^{1}\right)^{n},\left(\beta, 1^{n}\right)\right)$ via the localization formula:

$$
c \equiv i_{*} \frac{i^{*} c}{e_{T}(F)} \bmod \text { torsion }
$$

$\left(e_{T}(F)\right.$ is the equivariant Euler class) and one can compute fairly easily that:

$$
e_{T}\left(\bar{M}_{0, n}(X, \beta)\right)=\prod_{i=1}^{n} t_{i}\left(t_{i}-\psi_{i}\right)
$$

where $H^{*}(B T, \mathbf{Q})=\mathbf{Q}\left[t_{1}, \ldots, t_{n}\right]$.

One could conceivably use this idea to compute Gromov-Witten invariants, since all the components of $F$ are fiber products of lower-degree stable map spaces, and the Euler classes are always expressible in terms of the $t_{i}$ and $\psi_{i}$. But the combinatorial problem of enumerating the components of $F$ is a nightmare (see [13]). Instead, Kley and I use forgetful maps among these graph spaces:

$$
f: \bar{M}_{0,0}\left(X \times\left(\mathbf{P}^{1}\right)^{n+1},\left(\beta, 1^{n+1}\right)\right) \rightarrow \bar{M}_{0,0}\left(X \times\left(\mathbf{P}^{1}\right)^{n},\left(\beta, 1^{n}\right)\right)
$$

and

$$
g: \bar{M}_{0,0}\left(X \times\left(\mathbf{P}^{1}\right)^{n+1},\left(\beta, 1^{n+1}\right)\right) \rightarrow \bar{M}_{0,0}\left(\mathbf{P}^{1} \times \mathbf{P}^{1},(1,1)\right) \cong \mathbf{P}^{3}
$$


to cobble together a birational $T$-equivariant map:

$$
\Phi: M_{0,0}\left(X \times\left(\mathbf{P}^{1}\right)^{n+1},\left(\beta, 1^{n+1}\right)\right) \rightarrow \bar{M}_{0,0}\left(X \times\left(\mathbf{P}^{1}\right)^{n},\left(\beta, 1^{n}\right)\right) \times \mathbf{P}^{3}
$$

The advantage of this map is that the connected components of the total fixed locus $i^{\prime}: F^{\prime} \hookrightarrow M_{0,0}\left(X \times\left(\mathbf{P}^{1}\right)^{n+1},\left(\beta, 1^{n+1}\right)\right)$ lying in the preimage of $j: M_{0, n}(X, \beta) \hookrightarrow M_{0,0}\left(X \times\left(\mathbf{P}^{1}\right)^{n},\left(\beta, 1^{n}\right)\right) \times \mathbf{P}^{3}$ are not hard to enumerate. And from the more refined localization formula:

$$
\Phi_{*}\left(\frac{i^{\prime *} c}{e_{T}\left(F^{\prime}\right)}\right)=\frac{j^{*} \Phi_{*} c}{e_{T}\left(\bar{M}_{0, n}(X, \beta)\right.}=\frac{j^{*} \Phi_{*} c}{t_{1} t_{n+1}\left(t_{1}+t_{n+1}\right) \prod t_{i}\left(t_{i}-\psi_{i}\right)}
$$

we were able to deduce Theorem 3 , by further pushing forward to $X^{n}$ via $e v$ and choosing suitable equivariant Chern classes $c$ on the graph space.

This approach does have its limitations, however. It does not suggest a "reason" for the particularly nice organization of the relations in Theorem 3. In particular, the same approach yields many relations among $J$-functions for higher genus curves, which ought to be organized and analyzed in the context of the many conjectures about generating functions made up of higher-genus Gromov-Witten invariants. Perhaps the physicists can come to our rescue again.

\section{References}

[1] K. Behrend, Gromov-Witten invariants in algebraic geometry, Invent Math 127 (1997) 601-617.

[2] K. Behrend, The product formula for Gromov-Witten invariants, J Alg Geom 8 (1999) 529-541.

[3] K. Behrend and B. Fantechi, The intrinsic normal cone, Invent Math 128 (1997) 45-88.

[4] K. Behrend and Y. Manin, Stacks of stable maps and Gromov-Witten invariants, Duke Math J 85 (1996) 1-60.

[5] A. Bertram, Some applications of localization to enumerative problems, Michigan Math J 48 (2000) 65-75.

[6] A. Bertram, Another way to enumerate rational curves with torus actions, Invent Math 142 (2000) 487-512.

[7] A. Bertram and H. Kley, New recursions for genus-zero Gromov-Witten invariants, math.AG/0007082.

[8] P. Candelas, X. de la Ossa, P. Green and L. Parkes, A pair of Calabi-Yau manifolds as an exactly soluble superconformal field theory, Nuclear Phys. B 359 (1991) 21-74. 
[9] G. Ellingsrud and S. Stromme, Bott's formula and enumerative geometry, J. Amer Math Soc 9 (1996) 175-193.

[10] A. Gathmann, Absolute and relative Gromov-Witten invariants of very ample hypersurfaces, math.AG/9908054.

[11] A. Givental, Equivariant Gromov-Witten invariants, Int Math Res Notices 13 (1996) 613-663.

[12] T. Graber and R. Pandharipande, Localization of virtual classes, Invent Math 135 (1999), 487-518.

[13] M. Kontsevich, Enumeration of rational curves with torus actions, In: The moduli space of curves, (R. Dijkgraaf, C. Faber and G. van der Geer eds.) Prog in Math 129, Birkhäuser, Boston (1995) 335-368.

[14] M. Kontsevich and Y. Manin, Gromov-Witten classes, quantum cohomology, and enumerative geometry, Comm Math Physics 164 (1994), 525-562.

[15] Y.P. Lee, Quantum Lefschetz hyperplane theorem, math.AG/0003128.

[16] J. Li and G. Tian, Virtual moduli cycles and Gromov-Witten invariants of algebraic varieties, J Amer Math Soc 11 (1998) 119-174.

[17] B. Lian, K. Liu, and S.T. Yau, Mirror principle I, Asian J Math 1 (1997) 729-763. Mirror principle II, Asian J Math 3 (1999) 109-146. Mirror principle III, Asian J of Math 3 (1999) 771-800. Mirror principle IV, math.AG/0007104.

[18] Z. Ran, Enumerative geometry of singular plane curves, Invent Math 97 (1989) 447-465.

email: bertram@math.utah.edu 